\title{
DESCOBERTA DE MICROFLORA EM SEDIMENTOS DO GRUPO BAURU, CRETÁCEO DO ESTADO DE SÃO PAULO
}

\author{
Murilo Rodolfo de LIMA* \\ Sérgio MEZZALIRA** \\ Rodolfo DINO*** \\ Antonio Roberto SAAD****.
}

\begin{abstract}
RESUMO
A presença de fósseis animais (invertebrados e vertebrados) é relativamente comum nos sedimentos do Grupo Bauru. Contudo, o endemismo das faunas tem dificultado a obtenção de certas informações geológicas, entre as quais a datação precisa dos níveis portadores.

Por outro lado, a ocorrência de vegetais é muito mais rara. Merece, pois, destaque o registro de uma microflora bem preservada, procedente da Fazenda Nossa Senhora do Carmo, Município de São Carlos, Estado de São Paulo. Esta descoberta, asșociada à Litofácies Itaqueri, representa, na verdade, o primeiro encontro de palinomorfos no Cretáceo da Bacia do Paraná.

A associação, muito diversificada, conta com algumas espécies de valor estratigráfico, que permitiram atribuição de idade coniaciana. Tornou-se igualmente possível, através do seu estudo, efetuar algumas considerações de natureza estratigráfica, paleoecológica, paleoclimática e tectônica sobre o afloramento em questão.
\end{abstract}

\section{ABSTRACT}

The presence of fossil animals (invertebrates and vertebrates) in sediments of the Bauru Group is quite common. Despite this fact, the pronounced endemism of the faunas has not permitted, till now, the precise dating of the fossil-bearing levels.

On the other hand, the occurrence of plants is much scarcer. In this context, the discovery of a very well preserved microflora, at the Fazenda Nossa Senhora do Carmo, São Carlos Municipality, São Paulo States, deserves attention. This assemblage represents, in fact, the first record of palynomorphs in the Cretaceous of the Paraná Basin.

Study of the very diversified material, including several stratigraphically important species, has allowed the attribution of a Coniacian age for the fossiliferous strata. It has also been possible to drawn important stratigraphic, paleoecological, paleoclimatological and tectonic conclusions regarding the studied outcrop.

\section{INTRODUÇÃO}

O Grupo Bauru, representando, em termos amplos, o ciclo sedimentar cretáceo da Bacia do Paraná, ocupa grande parte do planalto ocidental do Estado de São Paulo, bem como importantes áreas dos Estados vizinhos (noroeste do Para- ná, sudeste de Mato Grosso, sudoeste de Minas Gerais e sul de Goiás). Embora bem estudado, ainda suscita grandes divergências entre os especialistas, especialmente no que se refere à sua subdivisão estratigráfica, datação e correlação.

Diversos campos de pesquisa têm-se empenhado no sentido de dirimir estas Brasil.

* Universidade de São Paulo - Instituto de Geociências - Caixa Postal 20899 - São Paulo, SP —

** Instituto Geológico - SMA - Caixa Postal 8772 - 01051 - São Paulo, SP - Brasil.

*** Cenpes - Cidade Universitária - Quadra 7 - Ilha do Fundão - Caixa Postal 809 - 21910 - Rio de Janeiro, RJ - Brasil.

**** IPT - Divisão de Minas e Geologia Aplicada - Caixa Postal 7141 - 05508 - São Paulo, SP Brasil. 
dúvidas. Dentre eles, destaca-se a $\mathrm{Pa}$ leontologia. É neste contexto que se integra o trabalho ora apresentado, que tem por objetivo divulgar a ocorrência de uma rica e bem preservada microflora. A partir do seu estudo, foi estabelecida uma idade mais precisa para o nível portador. Tornou-se igualmente possível tecer considerações acerca de seu significado paleoambiental, paleoclimático e paleogeográfico, contribuindo assim para melhor conhecimento desta unidade no Estado de São Paulo.

\section{ESTRATIGRAFIA}

Entre as diversas propostas de subdivịsão do Grupo Bauru no Estado de São Paulo destaca-se a de SOARES et alii (1980) pela sua relativa simplicidade e aplicabilidade. Embora não aceita por todos os autores, a coluna proposta considera a presença de apenas quatro unidades distintas, apresentadas a nível de formação, e uma quinta, classificada como litofácies. Estas são:

- Formação Caiuá - A unidade basal do grupo é constituída por arenitos de granulação fina a média, seleção regular a boa, escassa matriz argilosa, cores vermelho-arroxeadas e estratificações cruzadas de grande porte. De ocorrência geograficamente limitada à região do Pontal do Paranapanema, esta unidade estaria depositada discordantemente sobre o basalto, havendo registro de solos fósseis na sua base. Alguns autores, como VIEIRA (1980) e FREITAS (1982), discordam do enquadramento da unidade no Grupo Bauru, argumentando, o primeiro, que uma brusca mudança ambiental pós-Caiuá seria responsá=? vel pelo posicionamento da mesma junto a basaltos e sedimentos inferiores no Grupo São Bento, enquanto o segundo alega razões tecto-sedimentares. Outra. discordância de opinião freqüentemente manifestada diz respeito ao ambiente de deposição, interpretado como eólico por SOARES et alii (19.80) e lacustre por outros autores, entre os quais SUGUIO (1980).

- Formação Santo Anastácio Unidade imediatamente sobreposta à anterior, em contato gradacional, é constituída por arenitos muito finos a médios, castanho-avermelhados, estratificações cruzadas de baixo ângulo e acamamento horizontal, contando com raras intercalações lamíticas. Ocorre principalmente na porção sudoeste do Estado de São Paulo. Sua gênese, em ambiente fluvial meandrante, é bem aceita entre os especialistas.

- Formação Adamantina - Tratase da unidade mais complexa e melhor representada geograficamente em todo o Bauru. Abrange, pelo menos, quatro fácies distintas (Araçatuba, Taciba, Ubirajara e São José do Rio Preto), tratadas na literatura com diferentes enfoques (litofácies, membros, formações, etc.). O ponto em comum, que permitiu a SOARES et alii (op. cit.) englobá-las sob o nome Adamantina é a representação de ambientes fluviolacustres bastante organizados, através de bancos de arenito de granulação fina a muito fina, cor rósea a castanha, com estratificação cruzada, em alternância com bancos de lamitos, siltitos e arenitos lamíticos, maciços ou com acamamento paralelo.

- Formação Marília - A última unidade do Cretáceo da Bacia do Paraná é composta por arenitos grosseiros e conglomeráticos, ricos em feldspatos e minerais pesados, que ocorrem em bancos maciços ou com acamamento subparalelo. Camadas de lamitos avermelhados e calcários são também encontradas. Representa ambiente de deposição torrencial, com sedimentação de leques aluviais e lagos efêmeros.

Litofácies Itaqueri - Esta unidade é provavelmente a mais polêmica de toda a bacia. Está representada por arenitos grosseiros a conglomeráticos que ocorrem na borda da "Bacia" Bauru. Originalmente considerada como um conglomerado basal do grupo (ALMEIDA \& BARBOSA, 1953), foi posteriormente redefinida como uma unidade pósBauru, de idade mais recente (MEZZALIRA, 1974), ou como Bauru em posição estratigráfica não definida (BRANDT NETO et alii, 1980). Alguns autores, entre os quais FREITAS (1964), apesar de aceitarem o Itaqueri como conglomerado basal, propõem o abandono do termo, por não apresentar extensão geográfica. Finalmente, outro grupo de autores, incluindo SOARES et alii $(1973,1980)$, considera esta unidade como uma fácies lateral, não calcífera, associada preferencialmente ao topo da Formação Adamantina e sobretudo à 
Formação Marília.

\section{PALEONTOLOGIA}

A ocorrência de fósseis é conhecida no Grupo Bauru desde o início do século. CAMPOS (1978) menciona 73 localidades fossilíferas cadastradas, sendo o número atual certamente maior. Das localidades citadas acima, quatro dizem respeito à Formação Caiuá, sendo os fósseis representativos uma pinça de crustáceo e pegadas de tetrápodes.

Não há menção de fósseis para a Formação Santo Anastácio. STEIN (1980) faz referências à presença de orifícios nesta unidade, admitindo, contudo, que não foram encontradas evidências de que se tratem de tubos de vermes.

A grande maioria dos fósseis presentes no Grupo Bauru é procedente do topo da Formação Adamantina e base da Formação Marília (SOARES et alii, 1980). A lista de fósseis, bastante extensa, foi sumariada por MEZZALIRA (1974, 1980) incluindo representantes dos seguintes grupos:

\section{Invertebrados \\ Gastrópodes \\ Bivalves \\ Conchostráceos \\ Ostracodes}

\section{Vertebrados}

Restos de peixes (escamas, dentes)

Quelônios

Crocodilianos

Saurópodes

Terópodes

\section{Vegetais}

Oogônios de carófitas

Madeira de conífera

Restos vegetais indeterminados.

Vale a pena mencionar que os elementos dominantes em quase todas as associações fósseis do Grupo Bauru são os vertebrados, especialmente répteis. Das 73 localidades mencionadas anteriormente, apenas 3 incluem fósseis vegetais.

\section{DADOS PALINOLÓGICOS}

\subsection{Proveniência do material}

$\mathrm{O}$ afloramento de origem do material estudado pode ser considerado clássico na literatura. Acha-se em terras da antiga Fazenda Nossa Senhora do Carmo, situada $16 \mathrm{~km}$ a NE da cidade de São Carlos. Atualmente, a localidade é chamada Fazenda Nossa Senhora de Fátima. O primeiro registro desse afloramento foi feito por BJORNBERG et alii (1965), que citam a ocorrência de conglomerados silicificados na região. MEZZALIRA (1966) menciona a presença de fósseis, representados por uma fauna variada, composta por gastrópodes, bivalves, ostracodes e conchostráceos, além de fragmentos vegetais não identificados, equivocadamente referindo o afloramento como localizado na Fazenda Nossa Senhora Aparecida. O nível fossilífero é representado por siltito argiloso pardo-acinzentado, posicionando-se a cerca de $15 \mathrm{~m}$ acima do topo da Formação Serra Geral.

\subsection{Composição da microflora}

A microflora estudada, muito rica, é composta predominantemente por angiospermas, representadas por polens monocolpados, tricolpados, triporados, poliporados, hexaporotricolpados e tricolporados. Polens de gimnospermas estão também presentes, representados por tipos rimulados, inaperturados, sacados, monocolpados e poliplicados. A presença de esporos é também expressiva, destacando-se formas reticuladas e perisporadas.

Das quase cem formas presentes, muitas das quais novas (a nível específico ou genérico) e que integrarão trabalho de cunho sistemático em preparação, algumas possuem valor estratigráfico considerável. Entre os esporos, destacam-se Zlivisporis blanensis e Gabonisporis vigorouxii. Entre os polens de gimnospermas, são importantes Classopollis classoides, Araucariacites australis e Gnetaceaepollenites jansonii. Com referência aos polens de angiospermas destacam-se Tricolpites tienabaensis, Victorisporis roberti, Constantinisporis jacquei, cf. Accuratipollis sp., Cretacaeiporites polygonalis, Cretacaeiporites infrabaculatus, Hexaporotricolpites emelianovi, Confossia vulgaris e Tricesticillus americanus. 


\section{RESULTADOS}

\subsection{Idade}

A idade do Grupo Bauru não tem sido apresentada de forma conclusiva. Atualmente, o conteúdo faunístico, especialmente no que se refere aos dinossauros, parece posicionar a unidade, grosso modo, no Senoniano (MEZZALIRA, 1974). Entretanto, outros autores (MENDES \& PETRi, 1971) situam o Grupo Bauru, junto com as Formações Parecis e Urucuia, no Maestrictiano, embora admitam que a idade, de acordo com os répteis, é senoniana.

Evidências não paleontológicas também têm sido utilizadas para fins de datação. SOARES \& LANDIM (1975), baseados em estudo comparativo da evolução tectônica e sedimentar da parte norte da Bacia do Paraná com a Bacia de Santos, atribuem a idade da Formação Santo Anastácio ao final do Cretáceo Inferior (Aptiano?). Pela mesma razão, bem como pela contemporaneidade das rochas alcalinas da região do Triângulo Mineiro, os mesmos autores propõem idade cenomaniana a santoniana para a Formação Adamantina. Argumentos similares, inclusive soerguimento de arcos marginais, como a Serra do Mar e o Arco da Canastra, bem como idade do vulcanismo associado, entre 80 e 60 milhões de anos, levaram SOARES et alii (op. cit.) a atribuir idade compreendida entre - Santoniano e o Maestrictiano para a Formação Marília. Mais recentemente, a datação de lavas analcimíticas na Formação Adamantina levou COUTINHO et alii (1982) a suporem, com reservas, que parte da seqüência Bauru, portando inclusive restos de dinossauros, poderia ser de idade terciária (Paleoceno).

A presença das espécies acima mencionadas permite atribuir idade coniaciana para o afloramento de São Carlos. No local não ocorrem restos de dinossauros, o que levou MEZZALIRA $(1974,1980)$ a interpretá-lo como sendo relacionado à sedimentação cenozóica. A presença de arenitos silicificados, por um lado (BJORNBERG et alii, 1965), e de uma idade cretácea, ora definida, compatível com a idade senoniana fornecida pelos fósseis, por outro, altera substancialmente o quadro geral. O afloramento pertence muito provavelmente à Litofácies Itaqueri, a qual, por sua vez, deve ser incluída no Grupo Bauru. Dentro desta seqüência, parece bastante aceitável o ponto de vista exposto por SOARES et alii (1980, p. 183) de que o afloramento de São Carlos estaria relacionado à deposição da Formação Marília, representando tão-somente uma fácies não calcífera e argilosa.

\subsection{Paleoambiente}

Ausência completa de elementos do paleomicroplâncton indica deposição em condições seguramente não-marinhas. A associação inclui uma série de tipos de vegetais certamente viventes na região, entre os quais esporos perisporados e reticulados, associados a águas paradas. $\mathrm{O}$ ambiente provavelmente foi de pequenas lagoas.

A presença de polens dissacados, representando condições não tropicais, deve ser interpretada em função do maior transporte que sofrem estes grãos, originários provavelmente de regiões mais altas da Serra do Mar.

\subsection{Paleoclima}

O conjunto microflorístico é sugestivo de condições quentes, com exceção dos polens dissacados, cuja presença pode ser explicada, como foi mencionado anteriormente, através de transporte a partir de partes altas. A abundância e diversidade de esporos confirmam estas condições, sugerindo um microclima algo úmido. Contudo, a relativa abundância de polens (Gnetaceae-pollenites, Equisetosporites) indica que o clima da região, fora do sítio deposicional, era seco, possivelmente árido.

\subsection{Tectônica}

O nível de siltito estudado está intercalado em camadas conglomeráticas. Pela posição geográfica (borda de bacia), enquadra-se perfeitamente no esquema paleoambiental da Litofácies Itaqueri. Estariam, pois, preservados sedimentos de uma pequena lagoa, associada a leques aluviais representativos de taludes. Sequiência similar, portando a mesma microflora, ocorre do outro lado do arco marginal representado pela Serra do Mar, na Bacia de Santos 
(A.T.HASHIMOTO, comun. pessoal). É lícito portanto supor que os conglomerados da Litofácies Itaqueri estejam associados a falhas, possivelmente escalonadas, que por sua vez refletiriam um processo ascensional expressivo da Serra do Mar durante o Cretáceo Médio.

Assim, em um contexto mais amplo, a deposição de conglomerados associados aos sedimentos do Grupo Bauru, conforme observam FÚLFARO et alii
(1982), representaria uma conseqüência do processo de soerguimento da margem continental brasileira, produzida por amplos movimentos verticais, acompanhados de tectonismo local.

\section{AGRADECIMENTOS}

Ao Prof. Dr. Thomas R. Fairchild, os autores agradecem as críticas e valiosas sugestões.

\section{REFERÊNCIAS BIBLIOGRÁFICAS}

ALMEIDA, F.F.M. \& BARBOSA, O. 1953 Geologia das quadrículas de Piracicaba e Rio Claro, Estado de São Paulo. Rio de Janeiro, DNPM/DGM. 96p. (Boletim, 143).

BJORNBERG, A.J.S.; GANDOLFI, N.; PARAGUASSU, A.B. 1965 Ocorrência de conglomerados silicificados na região de São Carlos, SP. Ciência e Cultura, São Paulo, Supl., 17(2): 133.

BRANDT NETO, M.; COIMBRA, A.M.; BARELLI, N.; SPILLER, L.R. 1980 Sedimentos da Serra de Itaqueri (Formação Bauru e cobertura Cenozóica). In: MESAREDONDA SOBRE A FORMAÇÃO BAURU NO ESTADO DE SÃO PAULO E REGIÕES ADJACENTES. São Paulo, Sociedade Brasileira de Geologia, Núcleo São Paulo. p.63-68. (Publicação especial, 7).

CAMPOS, D.A. 1978 Carta geológica do Brasil ao Milionésimo: Folha de Paranapanema. Brasilia, DNPM. 84p.

COUTINHO, J.M.V.; COIMBRA, A.M.; BRANDT NETO, M.; ROCHA, G.A. 1982 Lavas alcalinas analcimíticas associadas ao Grupo Bauru (Kb) no Estado de São Paulo. In: CONGRESSO LATINO-AMERICANO DE GEOLOGIA, 5. ${ }^{\circ}$, Buenos Aires. Anais. Buenos Aires. v.2 p.185-196.

FREITAS, R.O. de 1964 Grupo Bauru. In: SĀO PAULO. INSTITUTO GEOGRÁFICO E GEOLÓGICO. Geologia do Estado de São Paulo. São Paulo, p.126-147. (Boletim, 41).

1982 Definição estratigráfica do Grupo Bauru por sedimentogramas. São Paulo, Instituto Geológico. 81p. (Boletim, 6).

FÚlFARO, V.J.; SAAD, A.R.; SANTOS, M.V.; VIANNA, R.B. 1982 Compartimentação e evolução tectônica da Bacia do Paraná. Revista Brasileira de Geociências, São Paulo, 12(4):590-610.

MENDES, J.C. \& PETRI, S. 1971 Geologia do Brasil. In: ENCICLOPÉDIA BRASILEIRA. Rio de Janeiro, Instituto Nacional do Livro. 207p, (Geologia, 9).

MEZZALIRA, S. 1966 Os fósseis do Estado de São Paulo. São Paulo, Instituto Geográfico e Geológico. 128p. (Boletim, 45).
MEZZALIRA, S. 1974 Contribuição ao conhecimento da estratigrafia e paleontologia do Arenito Bauru. São Paulo, Instituto Geográfico e Geológico. 163p. (Boletim, 51).

1980 Aspectos paleoecológicos da Formação Bauru. In: MESA-REDONDA SOBRE A FORMAÇÃO BAURU NO ESTADO DE SÃO PAULO E REGIÕES ADJACENTES. São Paulo, Sociedade Brasileira de Geologia, Núcleo São Paulo. pp.1-8. (Publicação especial, 7).

SOARES, P.C.; SINELLI, O.; PENALVA, F.; WERNICK, E.; SOUZA, A.; CASTRO, P.R.M. 1973 Geologia do Nordeste do Estado de São Paulo. In: CONGRESSO BRASILEIRO DE GEOLOGIA, 27. ${ }^{\circ}$, Aracaju, SE. Anais. Aracaju, Sociedade Brasileira de Geologia. v.1, p.209-229.

\& LANDIM, P.M.B. 1975 Comparison between the tectonic evolution of the intracratonic and marginal basins in South Brazil. Anais da Academia Brasileira de Ciências, Rio de Janeiro, 48(Supl.) p.313-324. ; FÚlFARO, V.J.; SOBREIRO NETO, A.F. 1980 Ensaio de caracterização estratigráfica do Cretáceo no Estado de São Paulo: Grupo Bauru. Revista Brasileira de Geociências, São Paulo, 10(3):177-185.

STEIN, D.P. 1980 Debate. In: MESAREDONDA SOBRE A FORMACৃÃO BAURU NO ESTADO DE SÃO PAULO E REGIŐES ADJACENTES. São Paulo, Sociedade Brasileira de Geologia. Núcleo São Paulo.p.47.(Publicação especial, 7).

SUGUIO, K. 1980 Fatores paleoambientais e paleoclimáticos e subdivisão estratigráfica do Grupo Bauru. In: MESA-REDONDA SOBRE A FORMAÇÃO BAURU NO ESTADO DE SÃO PAULO E REGIŌES ADJACENTES. São Paulo, Sociedade Brasileira de Geologia, Núcleo São Paulo. p.15-26. (Publicação especial, 7).

VIEIRA, P.C. 1980 Debate. In: MESAREDONDA SOBRE A FORMAÇÃO BAURU NO ESTADO DE SÃO PAULO E REGIÕES ADJACENTES. São Paulo, Sociedade Brasileira de Geologia, Núcleo São Paulo. p.93. (Publicação especial, 7). 